\title{
Stimuli-Controlled Manipulation of Synthetic Micrometre-Sized Vehicles for Bio-Inspired Fluidics ${ }^{\dagger}$
}

\author{
Larisa Florea *, Wayne Francis, Colm Delaney and Dermot Diamond \\ Insight Centre for Data Analytics, National Centre for Sensor Research, School of Chemical Science, Dublin \\ City University, Dublin 9, Dublin, Ireland; wayne.francis2@mail.dcu.ie (W.F.); colm.delaney@dcu.ie (C.D.); \\ dermot.diamond@dcu.ie (D.D.) \\ * Correspondence: larisa.florea@dcu.ie \\ + Presented at the 5th International Symposium on Sensor Science (I3S 2017), Barcelona, Spain, \\ 27-29 September 2017.
}

Published: 6 December 2017

Controlled movement in fluids is essential to the function of living systems. The desire of scientists to understand such complex motility in great detail, for over two centuries, has furthered our understanding of its intricacies. At the microscale, movement has been essential to the survival of all life, where individual cells can react to various chemicals by moving in a pre-programmed manner. This phenomenon is known as chemotaxis. To emulate such structures and processes, we present micro-vehicle droplets, based on ionic liquids (ILs) which show chemotactic behaviour. Such movement is generated through asymmetric release of an IL surfactant, which results in an imbalance in the local surface tension, causing the droplet to move spontaneously towards regions of higher surface tension. This behaviour can be exploited for programmed migration to specific locations in microfluidic devices, without the need for pumping or external stimulation of the system. Herein, we have developed signalling and seeking IL droplets, which chemotactically find each other in open fluidic networks by harnessing the chemical potential of their constituents and their environment. Additionally, we present chemotactic IL droplets which interact intimately with their fluidic system, offering the ability to make decisions, perform chemical reactions, carry out dynamic sensing-reporting and implement damage detection-repair. Such self-directed, multi-purpose movement of micro "vehicles" offers many intriguing opportunities in the microfluidics field. This could potentially stimulate novel research in droplet microfluidic devices, where the driving force for movement is dictated by the chemistry of the fluidic system itself, rather than through external control by the user. The realization of multifunctional biomimetic fluidic systems with advanced functionality, such as detection and repair of damage, self-management and healing could affect areas far beyond the frontiers of this research field.

(C) 2017 by the authors. Licensee MDPI, Basel, Switzerland. This article is an open access article distributed under the terms and conditions of the Creative Commons Attribution (CC BY) license (http://creativecommons.org/licenses/by/4.0/). 